\title{
Birke (Betula pendula): Mögliche Akklimatisierung an Ozon-Streß
}

\author{
Von Madeleine S. Günthardt-Golerg, R. Matyssek, Th, Kelier
}

\section{Einleitung}

Die Zelldifferenzierung und Organentwicklung entlang der Sproßachse läuft ab innerhalb der genetischen Rahmenprogramme. Während der Ausbildung modifizieren mikroklimatische und edaphische Faktoren die Merkmalsausprägung (TICHÀ 1985); es kann eine Akklimatisierung an diese Faktoren stattfinden. Solche Akklimatisierungen lassen die Pflanzen fortgesetzten Streß besser tolerieren. Findet auch eine Akklimatisiturung an eine chronische Ozonbelastung statt?

\section{Pflanzen und Methoden}

Vertopfte Birkenstecklinge (Betula pendula) eines örtlichen Mutterbaumes wurden vor dem Austreiben in die Freilandbegasungsanlage $20 \mathrm{Kammern}, 5$ pro Begasungskonzentration, 4 Bäume pro Kammer, gut gewässert und ernährt) eingestellt und bis zur Herbstverfärbung verschiedenen Ozonkonzentrationen ausgesetzt. Das Ozon wurde aus reinem Sauerstoff hergestellt und kontinuierlich der mit Aktivkohle gefilterter. Luft zugegeben (Konzentrationen: Kontrolle $\left.<0,003 ; 0,05 ; 0,075 ; 0,1 \mu \mathrm{I}^{-1}\right)$. 5 zusätzliche Pflanzen aus der Kontrollbehandlung wurden am 20. August in die $0,\left.1 \mu 1\right|^{-1}$-Ozonbegasung eingestellt (= nachträglich begaste Pflanzen). Die Blätter wurden numerierc, und aus den Zeirspannen bis zum Abschluß des Blattflächenwachstums sowie dem Auftreten visueller Schadsymptome wurde die jeweilige Ozondosis (Konzentration $\times$ Zeit) pro Einzelblatt berechnet. An 55 ausgewählten Blättern wurden mittels Lichtmikrostopie die stomatäre Dichte (Anzahl Spaltöffnungen pro $\mathrm{mm}^{2}$ ) und die Stärkereaktion (mit JJK), mittels Tieftemperaturrasterelektronenmikroskopie die Länge und der Öffnungszusrand der Schließzellen bestimmt. Gefrierquerbrüche wurden von 21 Blättern hergestellt (GÜNTHARDT-GOERG et al. 1992; SCHEIDEGGER et al. 1991).

\section{Ergebnisse}

Die Flächen der Birkenblätter blieben reduziert, wenn die Ozondosis (Ozonkonzentration $\times$ Zeitdauer der Blattentfaltung) mehr als $26 \mu \mathrm{l}^{-1} \mathrm{~h}$ betrug (Abb. $1 \mathrm{~A}$, weitere morphologische Parameter s. GünthardT-Goerg et al. 1993). Mit abnehmender Blattfläche (bei $0,1 \mu \mathrm{l}^{-1}$ Ozon auch reduzierte Zellgröße, Abb. 2: lange Säulen) nahm die Spaltöffnungsdichte zu (Abb. 1B). Die Öffnungsweite der Schließzellen war in Blättern ohne oder mit geringen visuellen Schadsymptomen unter Ozon zu verschiedencn Zeitpunkten enger als unter Filterluft (Abb.2, kurze Säulen). Die bis zur Ausprägung visueller Blattschadsymptome erforderliche Ozondosis (Abb. 3) nahm mit steigender Ozonkonzentration zu.

Unter Filterluft ausgebildete Blätter an nachträglich begasten Kontroll-Bäumen fielen bei niedrigerer Ozondosis $a b$, als es bei unter Ozon ausdifferenzierten Blättern der Fall war (Abb.3, schwarze Säulen). Nachträglich unter Ozon ausgebildete Blätter dieser Bäume 
$A b b, 1$. Blattfläche (A) und Anzahl Spaltöffnungen pro $\mathrm{mm}^{2}(B)$ in Abhängigkeit von der Ozondosis (Konzentration $\times$ Zeit) während des Blattwachstums. Blätter an nachträglich begasten Kontroll-Bäumen (ausgefüllte Symbole) sind entweder unter Filterluft oder unter Ozon ausgebildet

Fig. l. Leaf area $(A)$ and stomatal density $(B)$ in relation to the ozone dose (concentration $\times$ time) during leaf extension growth. Leaves from transferred control trees (filled symbols) were either formed in filtered air or newly formed under ozone after the transfer

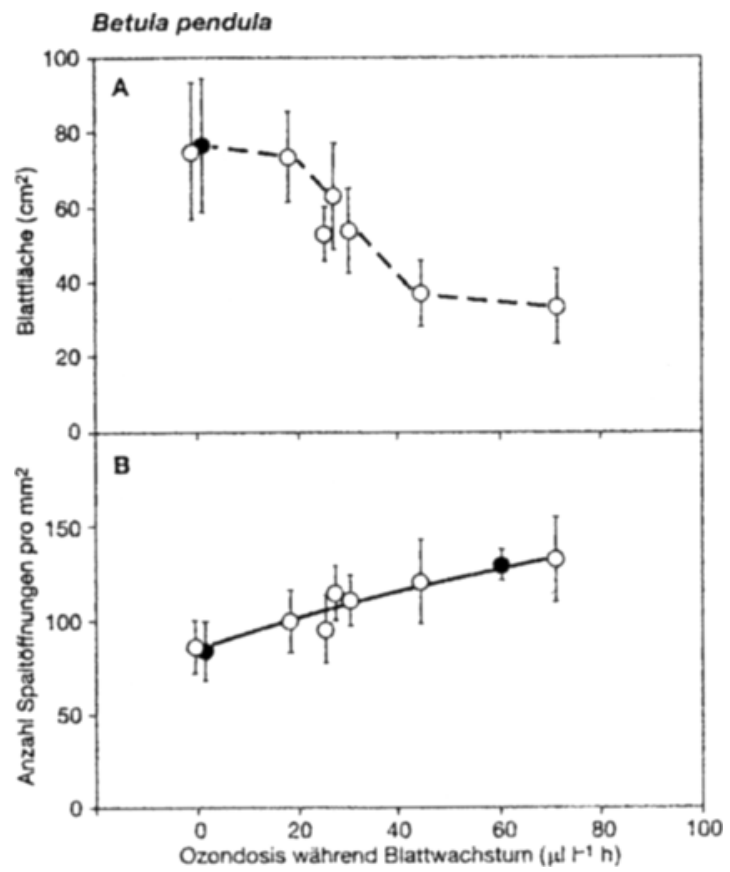

zeigten jedoch eine dosisabhängig erhöhte Spaltöfnungsdichte (Abb. IB, schwarze Symbole), kleinere Sclnließzellen und verengte Spaltöfnungswiten (Abb. 2, 0,1\%). Dic zur Ausprägung von Schadsymptomen nötige Ozondosis (Abb. 3, 0,1*, Säulen mit Raster) war an diesen Blättern gleich derjenigen der $0,05 \mathrm{ul}^{-1}$-Begasung.

Bei begasten Blättern mit ersten visuellen Schadsymptomen wurden mittels Tieftemperaturrasterelektronenmikroskopie "nicht wäßrige tropfenförmige Exsudate (unetchable droplet-like exudates, sehr wahrscheinlich Zellwandmaterial) beobachtet (GüNTHARDTGoerg et al. 1993). Zuerst traten die Exsudate an den Zellwänden der Mesophyllzellen rund um die Stomahöhlen auf der Blartunterseite (Blattoberseite ohne Stomata) auf und breiteten sich bis zu den Palisadenzellen aus. Schließlich kollabierten einzelne oder Gruppen von Mesophyllzellen. Nach ihnen folgten die Epidermiszellen und als letzte die Spaltöffnungen, nachdem sie sich bei kollabierten Nachbarzellen vorübergehend passiv weit geöffnet hatten.

Mit zunehmender Schädigung der Mesophyllzellen wurde weniger Assimilacionsstärke (Amylose) beobachtet, welche zudem nur bis zu den Bündelscheiden abtransportiert wurde und dort wiederum als Stärke liegenblieb. Die verengten Schließzzelien waren mit Stärke (Amylopektin) vollgepackt, und parallel dazu traten Amylopektinkörner in den nicht assimilierenden Epidermiszellen auf. Unter Filterluft ausgebildete Blätter zeigten bei nachträglicher Ozonbegasung stark reduzierte Assimilationsstärke, aber keine der oben. genannten Stärkemuster.

\section{Diskussion}

In voll ausgebildeten mesophytischen Blärtern dringt Ozon durch die Spaltöffnungen ein und zerfällt rasch im Apoplast (LAISK 1988), wo in der Folge wahrscheinlich Radikalkettenreaktionen zur Zellschädigung führen. Chronische, geringe Ozonkonzentrationen wirken schon während der Blattdifferenzierung (Abb. 1 und 2, Dosisabhängigkeit). Je 


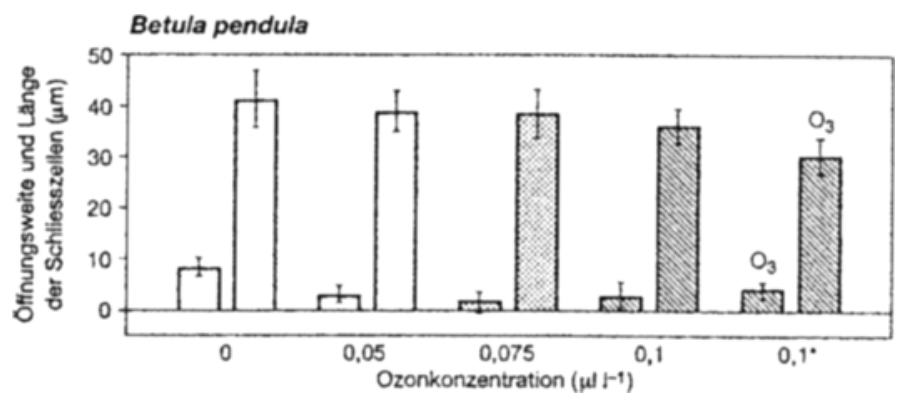

Abb. 2. Mittlere Öffnungsweite (kurze Säulen, Mittelwerte signifikant verschieden) und mittlere Länge (lange Säulen, nur bei $0,1 \mu l \mathrm{l}^{-1}$ Ozon signifikant verschieden von der Kontrolle, $t$-Test) der Schließzellen unter verschiedenen Ozonkonzentrationen (Beispiel von Blättern gleichen Alters am 27. September 1989 , Erntezeitpunkt 8.30 Uhr, $17,6^{\circ} \mathrm{C}, 63 \%$ relative Feuchte)

Signuturen (Abbildung 2 und 3): $\mathrm{F}=$ unter Filterluft, $\mathrm{O}_{3}=$ unter Ozon ausgebildete Blätter an nachträglich mit Ozon $\left(0,1^{*}\right)$ begasten Kontrollptlanzen. Visuelle Blattsymptome: $\square$ grün; $\square$ einzelne hellgrüne Punkte; $\square$ hellgrüne Punkte auf der ganzen Blattfläche oder bronzefarbiges Blatrgrün oder winzige schwarze Punkte; $\square$ kleine Nekrosepunkte; $\mathbf{a b g e f a l l e n e ~ B l a ̈ t t e r ~}$

Fig. 2. Mean aperture (small bars, all significantly different from control) and mean length (long bars, different from control at $0.1 \mu \mathrm{l}^{-1}$, t-test) of guard cells under different ozone concentrations (harvest September 27 , at $8.30 \mathrm{a} . \mathrm{m}, 17.6^{\circ} \mathrm{C}$ and $63 \%$ relative humidity, leaves of same age)

Signatures (Figure 2 and 3 ): $F=$ leaves formed in filtered air, $\mathrm{O}_{3}=$ leaves formed in ozonized air on control plants that had been transferred from filtered air to $0.1 \mu \mathrm{ll} \mathrm{l}^{-1}$ ozone $\left(0.1^{\%}\right)$ on August 20. Macroscopical leaf symptomes: $\square$ green; $\square$ isolated light green dots; $\square$ light green or bronze-green leaf colour, tiny biack dors; $\square$ small necroric spots; $\mathbf{D}$ shed leaves

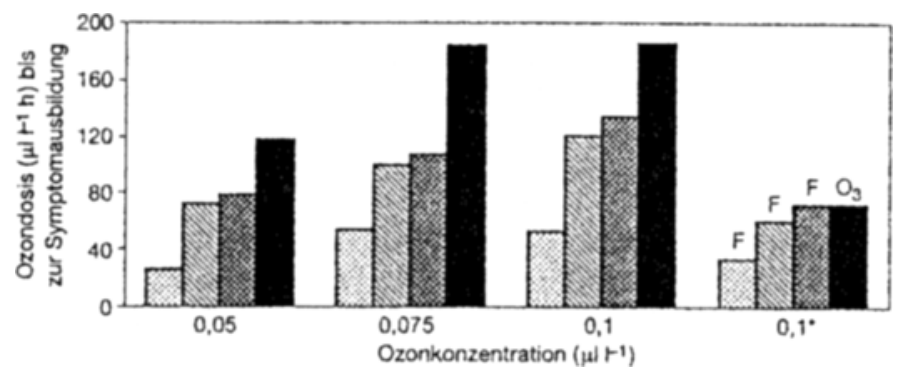

Abb. 3. Mitrlere Ozondosis bis zum Auftreten visueller Symprome in Abhängigkeit von der Begasungskonzentration

Fig. 3. Mean ozone dose causing the appearance of macroscopical leaf symptoms in relation to the fumigation with different ozone concentrations

langsamer die Blätter austreiben, desto höher ist die Ozondosis während der Differenzierung. Unter Ozon ausgebildete Blätter sind in der Folge auf fortgesetzte Ozonbehandlung weniger empfindlich, während nachträglich begaste Kontrollpflanzen baldigen Blattfall aufweisen (Abb.3). Man kann deshalb von einer Akklimatisierung an Ozon sprechen, deren strukturelle Differenzierung Ähnlichkeit mit derjenigen unter Wasserstreß zeigt. Die Mechanismen der Blatrdifferenzierung unter Ozonstreß sind bislang unbekannt.

Eine Akklimatisierung kann sich auch in der Verengung der Stomata widerspiegeln: Gasaustausch und somit auch die Schadstoftautnahme sind verringert. Bei langsam fortschreitender Schädigung beginnen einzelne Mesophylizellen zu kollabieren, während die Spaltöffnungen die $\mathrm{CO}_{2}$-Versorgung der verbleibenden Mesophyllzellen nicht limitieren. Der Mesophyllkollaps verschlechtert die Bilanz aus Kohlenstoffgewinn pro Wasserverbrauch (MATYSSEK et al. 1991). Die Stärkeverteilung zwischen Spaltöffnungen, Mesophyll- 
zellen und Transportbahnen verändert sich. Die Stärkeansammlung in den Spaltöffnungen könnte ein Anzeichen der Verengung sein. Die entlang der kleinen Blattnerven angehäufre Stärke steht vielleicht mit der veränderten Assimilatverteilung in der ganzen Pflanze im Zusammenhang (reduziertes Wurzel/Sproßverhältnis, MATYSSEK et al. 1992).

Eine Akklimatisierung in höheren Lagen geschieht möglicherweise nicht nur an die Temperatur und den Wasserstreß, sondern auch an die kontinuierlich erhöhte Ozonkonzentration. Es ist zu prüfen, welchen Eintluß das Ozon auf die Hormone oder „hormonähnliche Metaboliten" hat, die in den Prozessen der Blattdifferenzierung, Spaltöffnungsregulation und Kohlenstoffverteilung mitwirken.

\section{Zusammenfassung}

Durch Ozonbegasung in einem Birkenklon hervorgerufene Änderungen der Blattfläche, der stomatären Dichte, der Länge und des Öfnungszustandes der Stomata sowie der Stärkeverteilung werden vorgestellt und hinsichtlich einer möglichen Akklimatisierung an Ozon diskutiert.

\section{Summary}

Birch (Betula pendula): possible acclimatization to ozone-stress

Leaf area change, stomatal density, length and width of the guard cells, and starch distribution in one birch clone exposed to ozone are presented and discussed with regard to possible acclimation to ozone.

\section{Literatur}

Günthardt-Goerg, Madeleine S.; Matyssek, R.; Scheidegger, C.; Keller, T., 1993: Differentiation and structural decline in the leaves and bark of birch (Betula pendula) under low ozone concentrations. Trees 7, 104-114.

LaISK, A.; Kull, O.; Moldat, H., 1989: Ozone concentration in intercellular spaces is close to zero. Plant physiol. 90, 1163-1167.

Matyssek, R.; Gürthardt-Goerc, Madel.eine S.; Keiller, T.; Scheidegcer, C., 1991: Impairment of gas exchange and structure in birch leaves (Betula pendula) caused by low ozone concentrations. Trees 5, 5-13.

Matyssek, R.; Günthardt-Goerg, Madeleine S.; Saurer, M.; Keller, T., 1992: Seasonal growth, $\delta^{13} \mathrm{C}$ in leaves and stem, and phloem structure of birch (Betula pendula) under low ozone concentrations. Trees 6, 69-76.

Scheidfgger, C.; Günthardt-Gofrg, Madelfine S.; Matyssek, R.; Hatvani, P., 1991: Lowtemperature scanning electron microscopy of birch leaves after exposure to ozone. J. Microsc. (Oxford) $161,85-95$.

TiCrA, INGRID, 1985: Onthogeny of leaf morphology and anatomy. In: Photosynthesis during leaf development. Task for vegetation science 11. Hrsg. Sestac, Z. Dordrecht: Junk, S.16-50.

Anschrift der Autoren: Dr. Madelejine S. Güntrardt-Goerg, Dr. R. Matyssek, Dr. Th. Keller, Eidgenössische Forschungsanstalt für Wald, Schnee und Landschaft, Zürcherstraße 111, CH-8903-Birmensdorf, Schweiz 